Chapter 3

\title{
Synthesis of Flow Simulation Methods for Fast and Accurate Gas Turbine Engine Performance Estimation
}

Ioannis Templalexis

Additional information is available at the end of the chapter

http://dx.doi.org/10.5772/54411

\section{Introduction}

Gas turbine engine development and maintenance comprises a great amount of risk. Nowadays a company on its own, the engine manufacturer for instance, cannot afford the entire engine development risk. At the same time, manufacturers need to provide their customers, the engine users, with a competitive maintenance package. Investments on gas turbine engine development and maintenance were magnified over the years, a process driven both by the competition and strict airworthiness and environmental regulations. Consequently, manufacturers are looking both for risk share partners and cost shrinkage.

A principal tool to achieve the aforementioned goals is computer based, engine performance simulation. Risk share partners need to have a view, or better to say, an evidence regarding the performance of the engine under development. Gas turbine engine performance simulation however, has a much greater impact on narrowing down the engine development related cost and on providing early evidence of engine malfunction, thus suppressing also the maintenance costs.

Computer based gas turbine engine performance simulation and the derived methods are classified and selected for a particular application, based on the leverage between accuracy and computational load. On one end of the classification scale stand the zero dimensional (0D) methods and on the opposite end stand the so called Computational Fluid Dynamics (CFD) methods based on Reynolds Averaged Navier Stokes (RANS) equations.

The current chapter aims to present a gas turbine engine, tailor made, performance simulation tool that stands out as an optimum combination of accuracy and execution cost. The cost of applying a certain simulation method rises with computational load. The architecture of the simulation tool under context is justified and at the same time it takes advantage of the fact 
that the prescribed accuracy for the simulation of each component is not the same. Consequently a single flow simulation tool, when applied on the entire engine would eventually cover the prescribed requirements for simulation of certain components but not of the entire engine. The simulation methodology that is to be presented over the following paragraphs, considers variable dimensions regarding the flow resolution of each component in order to address firmly gas turbine engine simulation requirements.

The current chapter, consists of two major sections. The first one addresses the simulation tools and methods for each component. The second section describes the amalgamation of these methods and tools to give the integrated gas turbine engine simulation tool. Before that a brief literature review will be given regarding similar efforts.

\section{Literature review}

Gas turbine engine simulation has been addressed as an issue by several researchers during the past. The current section aims to list an indicative sample of relevant research work and associated software. The author will not go into details, however readers that are further interested on this specific research field, are advised to address to the citations given below. Joachim Kurzke [1], has been very active on gas turbine engine simulation, specialized on 0D flow simulation and component map generation. Also M.G. Turner et al [2] have dealt with gas turbine engine simulation, producing hybrid tools aiming to reduce computational load, while retaining an acceptable accuracy level. Alexiou et al [3], [4], have also been very active in the research field of engine flow simulation Additionally Alan Hale et al [5], have developed hybrid tools for effective gas turbine engine simulation. Finally the author of the current chapter working in conjunction with Cranfield University research team and in particular with Dr. Vasilios Pachidis has contributed substantially to the research field under context [6], [7], [8]. Very little gas turbine engine simulation software has been developed up to the moment capable of capturing the engine performance alternation under uneven inlet flow conditions. Several flow calculation methods are available in the open literature, but the computational resources required, for the system of equations to be solved numerically throughout the gas path, are enormous. Such a code is the main outcome from the Numerical Propulsion System Simulations (NPSS) project realized at the NASA Glenn Research Center (GRC). A high fidelity 3-D gas turbine performance code has been developed for gas turbine engine simulation. In NASA report [9] a case study of the CF-90 high by-pass ratio is presented.

\section{Fields of application for gas turbine engine simulation tools}

As it was mentioned in the introductory section, there exists a very wide range of flow simulation packages that are tailored to, or can be applied for, gas turbine engine performance simulation. A vast categorization of these methods and derived tools, is usually made on the basis of the number of dimensions considered for the flow analysis. Alternatively we very 
often see those methods to be categorized according to the level of flow complexity considered. In specific, according to the former categorization we have:

i. Zero Dimensional (0-D)

ii. $\quad$ One Dimensional (1-D)

iii. Two Dimensional (2-D)

iv. Three Dimensional (3-D)

according to the later categorization we have:

i. RANS

ii. $\quad$ Euler based

iii. Potential flow based

Flow simulation methods and tools out of all the aforementioned categories could potentially be applied along a certain sector of the engine development road. On figure 1 the range of application for a certain category, is shown as a vast proportion of the entire development road of the engine, starting from the initial specifications up to the engine testing. Ideally the 3-D high fidelity methods could be applied during the entire development period of the engine. However their application range as it can be seen on Fig. 1 is restricted to the right end. That is just before the final manufacturing drawings are sent for metal cutting. The increased simulation fidelity of these tools comes with an increased investment and operational cost on computational power. Several hours or even days in some cases stand in between setting the inputs and obtaining a converged solution.

It remains questionable whether the increasingly available computational power at a lower cost will shrink the convergence time since more and more sophisticated turbulence and viscosity models appear to give a more accurate flow solution at the expense of the excess computational power available. As a result of the above, the usage of high fidelity 3-D flow simulation tools can be economically justified when applied to the final component or engine design. The aim is to trim the final design in order to match the closest possible the pre-defined performance. What should be avoided at all costs is to be obliged to re-design and re-fabricate a part or the entire component as a result of the fact that the experimental results do not match to the prescribed requirements. Consequently high fidelity simulation is welcomed, but it should only be used when such high fidelity results are absolutely necessary.

On the other hand, focusing on the left end (always according to Fig. 1) a narrow application range of $0-\mathrm{D}$ and 1-D methods is spotted. That is actually during the preliminary design phase, when a wide range of a large number of design parameters are still under discussion resulting in a large amount of simulation cases to be examined. At this stage, qualitative assessment is of primary interest as opposed to quantitative assessment that becomes increasingly important during the following development phases. Low fidelity - low computational load methods match perfectly to the requirements of the preliminary design phase. 


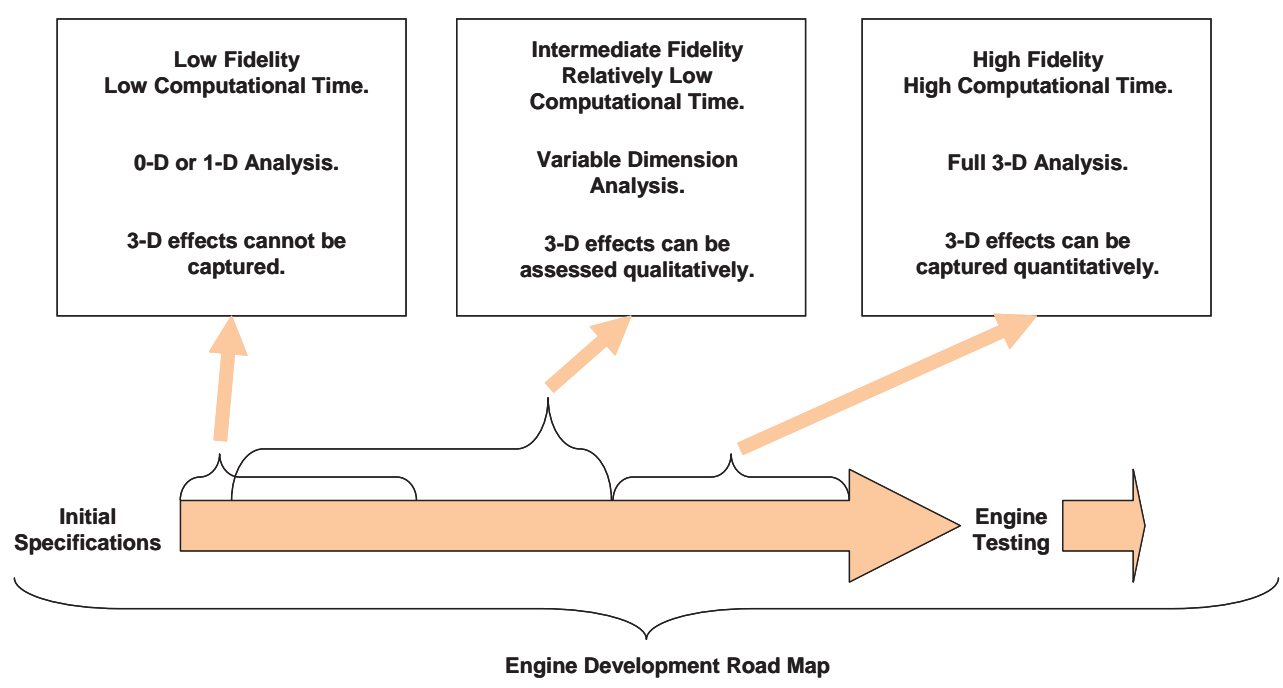

Figure 1. Field of Application for Gas Turbine Engine Simulation Tools

In between low fidelity and high fidelity methods and derived tools, stand the hybrid flow simulation methods that could be identified as intermediate fidelity - relatively low computational time methods. Attempting to underline the identifying characteristic of these methods one would conclude that the required computational time before convergence is not an important issue to consider. In other words the number of case studies is not determined by computational load restrictions. Requiring the minimum possible computational time consumption while demanding an increased fidelity of the derived results, those methods end up having the following characteristics:

i. $\quad$ They usually are 2-D methods, treating the flow as in-viscid. A 3-D method would be accepted when solving the flow as in-viscid and irrotational.

ii. Component performance disturbances due to 2-D and even 3-D non-uniformities are at a certain accuracy level captured and assessed through hybrid simulation tools applying different simulation methods between components.

iii. Performance information encoded in experimental results or in CFD results is adhered by these simulation tools in a tabular format (Component performance map) or even better in the form of empirical equations.

Based on the above mentioned characteristics of these methods and as far as their application range is concerned, intermediate fidelity methods are continuously expanding towards both ends of the engine development trail. It is not surprising that nowadays these methods are gaining increasing attention by engine manufacturers and research institutes. It is the author's view that given the cost reduction of computational power such methods will completely cover the range of applications of 1-D and 0-D methods. The fraction of the application field of the 
high fidelity methods that can be covered by these methods depends on the ability of a specific tool to adopt experimental results.

\section{Components of the hybrid simulation method}

The current section explicitly refers to the components of the hybrid simulation method. More specifically it refers:

1. To the method and derived tools for gas turbine engine intake simulation, where the flow is considered as potential. The simulation tool is based on the Vortex Lattice Method (VLM).

2. To the methods and derived tools for the flow simulation of the most sensitive engine component, the compressor. The two modules of the proposed flow simulation strategy, are based on "multi parallel compressor method" and "Streamline Curvature Method" respectively.

3. To the method and derived tools for the flow simulation of the remaining engine components (Combustion chamber, turbine, nozzle). The performance simulation tool is based on the 0 -D performance simulation method.

The architecture of the described Intake - Gas Turbine Variable Dimension Performance Simulation Method, will be explained during the second main section of the current chapter. It will then be more clear to the reader the reasons for selecting a specific method for each component as detailed to the three points above.

\subsection{Intake section}

\subsubsection{Intake flow simulation method}

The flow within the engine intake is treated as in-viscid. The three dimensional flow nonuniformities are to be carried through the intake up to the compressor face, while keeping the computational load to the minimum possible. A flow simulation method that meets the above mentioned pair of requirements is the VLM. The VLM was among the earliest of such methods written in computer code, mostly addressed for the computation of airfoil aerodynamic characteristics. It was first conceived at the late 30's, but it could not be applied efficiently until the early 60 's. The reason was that being a purely numerical method involving big matrix inversions, it had to wait for the computers to develop sufficiently in order to support such calculation load [10].

It belongs as a method to the group of Panel Methods (PM) since:

- The linear potential flow equation is solved.

- Panels are used for the description of the geometry.

- Singularities are placed on a surface. 
- The "Neumann" boundary condition is applied to a number of control points.

- A system of algebraic equations is solved to determine the singularities strength.

What makes the VLM a separate method are the facts that:

- It is usually applied on lifting surfaces.

- One kind of singularity (vortex filaments) is strictly used. It is not distributed over the entire surface, but only along the surface boundaries.

According to the VLM the flow field around a lifting surface is established by superimposing the free stream flow velocity to the velocities induced by the vortex filaments. It is reminded that vortex filaments have constant circulation $\Gamma$ along a certain vertex line which cannot begin or end abruptly in a fluid. It must either be closed, extend to infinity or end at a solid boundary. The circulation about any section is the vortex strength. A vortex obeys to the Biot-Savart law and according to its specific shape it has a specific mathematical expression for expressing the induced velocity, at any arbitrary point in space. [11]. It is reminded that the Biot-Savart law was initially defined for the description of the magnetic flow field induced around a current carrying conductor. It is also used for the determination of the velocity vector field appearing around a vortex filament, due to the obvious similarity between the two physical phenomena. For a general 3-D vortex filament (see Figure 2) the velocity increment induced at an arbitrary point in space $(\mathrm{P})$, by an infinitesimal vortex segment $(\mathrm{dl})$ is given by the following equation:

$$
\mathrm{dV}_{\mathrm{p}}=\frac{\Gamma}{4 \cdot \pi} \cdot \frac{\mathrm{dl} \times \mathrm{r}_{\mathrm{dl}, \mathrm{p}}}{\mathrm{r}_{\mathrm{dl}, \mathrm{p}}{ }^{3}}
$$

Where $\Gamma$ the circulation around the filament (constant).

Hence the velocity at point $\mathrm{P}$ due to the entire vortex filament would be:

$$
\mathrm{V}_{\mathrm{p}}=\frac{\Gamma}{4 \cdot \pi} \cdot \int \frac{\mathrm{dl} \times \mathrm{r}_{\mathrm{dl}, \mathrm{r}}}{\mathrm{r}_{\mathrm{dl}, \mathrm{r}}{ }^{3}}
$$

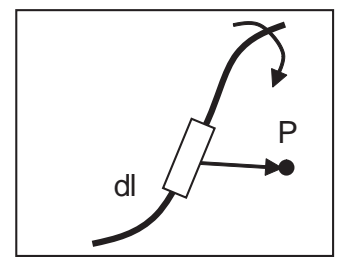

Figure 2. Vortex filament.

The evaluation of the above integral is not always a simple straightforward calculation procedure. The vortex filaments that are used as "construction elements" for the VLM have a well defined and documented solution as they have been used extensively during the past. Vortex filament types can be combined to give new singularity elements ready to be used by 
VLM calculation schemes, just like the source and the sink when combined together give a doublet. The most widely used "synthesized" vortex is the horseshoe vortex. It consists of three straight line vortices. One finite length vortex and two semi-infinite vortices. It is basically the model of a finite wing. The two semi-infinite vortices represent the wing tip vortices and the finite vortex represents the wing span. According to the literature review that has been done the numerical schemes using purely vortex singularities can all be classified into two major categories. In the first category horseshoe kind of vortex filaments are incorporated and in the second category the closed bound vortex filaments around the panel surfaces are used.

A horseshoe vortex method would not be attractive for the intake internal flow simulation. Consequently the only candidate left is the "closed vortex filaments" method [11], having more advantages when compared to the rest of the flow simulation methods, for the development of a rapid calculation scheme to be applied in the intake internal flow regime. Both the flow around lifting and non lifting surfaces can be equally well simulated. Various alternative solutions for the wake handling can be incorporated while very little computer resources are required for convergence.

In particular the VLM used for the intake flow simulation is summarized by the following application steps. Firstly the geometry is defined using an arbitrary number of panels in the sense that there is not any upper limit other than the computer processor capabilities. A sensitivity analysis concerning the optimum number of panels to be used fits perfectly at this point. The panels are flat of triangular or rectangular shape. Their control points coincide with their geometrical centers, where a local Cartesian coordinate system is being defined by a normal and two tangential vectors. Secondly a closed vortex filament distribution is assumed over the surface surrounding the panel boundaries. The "Neumann" boundary condition is applied resulting in a linear system of $\mathrm{n} x \mathrm{n}$ independent equations, where $\mathrm{n}$ is the number of panels. The solution of this system gives the vortex strength matrix. The Kutta - Jukofski boundary condition is applied at the panel trailing edges where wake is expected to be developed. The wake is left free to relax according to the induced velocities. The flow vector field is continuously updated through the iterative process, by the velocity components induced from the wake panels generated. The convergence criterion is based on the aerodynamic forces induced on the panels.

\subsubsection{Intake flow simulation software description}

The intake flow simulation software, as any 3-D flow simulation software consists of the three following main modules:

- Pre-possessing which encounters the geometry definition and the solution grid settings where applicable.

- Processing, or in other words the flow solver. The module that derives the mathematical solution.

- Post-possessing, which refers to the way the developer chooses to present the results calculated by the above mentioned module. 
Developers of 3D flow simulation software, despite the fact they think in terms of the three distinct modules mentioned above, these modules are not always distinguishable by the end user. The pre-processing module should ask and acquire from the user in a certain numerical format the set of geometrical data that lead to the geometry related to the flow simulation regime (it is irrelevant whether intermediate calculations take place). The pre-processor of the VLM tool under context is used to create the intake geometry covered in panels. It is one of the input files fed into the processing unit.

The processor receives the input files, conducts the calculations and delivers the set of output files for post processing. It is the core of a CFD software. An integral solver does not contain only the set of equations ready to be solved, but also a set of subroutines in order to reassure convergence, under any set of inputs. Additionally the solver should ideally inform and consult the user during execution.

The post processing of the results is not necessarily done, although it is very convenient, by the same software that conducts the pre-processing is also highly dependent upon the needs of a case study. The VLM software as a post-processor offers only the visualization of streamlines.

\subsection{Compressor section}

\subsubsection{Compressor flow simulation method}

Attempting to classify gas turbine engine components based on the flow regime in terms of complexity, temperature and pressure, three major groups can be recognized: The components located upstream of the combustion chamber, the components located downstream of the combustion chamber and the combustion chamber itself. Within the "upstream" components, the pressure is rising in the direction of the flow, whereas the opposite takes place along the "downstream" components. Upstream of the combustion chamber due to the "un-physical" character of the process, since the air is forced to move against an adverse pressure gradient, the flow is becoming very complex and often unpredictable. This is even more pronounced in the compressor, as it consists of both rotating and non-rotating parts. The flow within the combustion chamber is extremely complex, as it is actually controlled by the thermodynamics and chemistry of the combustion process. Finally, within the working medium expansion region of the engine, the flow can be considered relatively simple from the fluid mechanics point of view.

It is therefore evident that the most critical component of the gas turbine engine regarding its operation and its response to the changing operating conditions is the compressor. Consequently the compressor becomes very challenging, when it comes to the flow simulation under the requirement for minimum computational power consumption. Flow non-uniformities conveyed through the engine intake down to the engine face affect severely the compressor overall performance and operational stability. The later refers to the minimum flow that can be handled before surge occurs. Therefore the compressor response should be known throughout its operational envelope, for all possible inlet conditions and throttle schedules. Using a single flow simulation method to assess the compressor performance would unavoidably lead 
to a 3-D tool. Euler or RANS based methods are excluded when referring to fast assessment tools. On the other hand VLM cannot cope with the flow regime present in a compressor as it is highly turbulent and 3-D boundary layers are developed especially at the rear stages. Additionally the application of VLM method in the case of a multistage compressor simulation demonstrates several geometry and convergence handling problems, where the wake handling problem stands above all. This problem arises only in the case where one blade row is immediately followed by a second one (rotor stator). Even in the case where the stator wake passes smoothly through the rotor during the first iterations without causing any instabilities, a few iterations later, when the rotor would have rotated by 60 degrees for example, the wake twists un-physically, remaining trapped between the rotor blades it first crossed. The physical process dictates that the following row truncates the wake generated by the blades of the upstream blade row. Attempting to program the solver to do so, the phenomenon cannot still be simulated very accurately because it is impossible for the wake to be cut down to less than one panel. The axial distance between blade rows in modern compressors is less than a wake panel's length. On the top of that, wake is dissipated as it travels down between the moving and stationary blades and it is not conserved as in the case of Panel Methods.

Consequently the 3-D flow profile present at the compressor inlet plane has to be de-composed and let each component be treated by a separate tool. In the case presented herein, the profile is decomposed to a radial and a circumferential component. The former is treated with a streamline curvature method based tool and the later is treated with a multiparallel compressor method based tool.

\subsubsection{Streamline curvature flow simulation method}

The origin of the SLC method, before even being identified as a separate calculation method, lies on Wu's through-flow theory [12]. The method under its current name was developed independently by Smith [13] and Novak [14] in the United States and Silvester and Hetherighon [15] in the United Kingdom. However, at that time the solutions were still restricted to the duct regions. The basic idea lies on the integration of the full radial equilibrium equation across the blade edges in conjunction with the flow continuity equation, for the determination of the meridional velocity profile across the compressor. Frost [16] took the method a step further, by applying it within the blade rows, demonstrating thus the first SLC-method-based flow representation within a compressor. Finally Senoo and Nakese [17] and Novak and Hearsey [18] reported quasi-3D SLC method applications.

During the approximately forty years of existence of the SLC method, numerous authors have proposed several variations of the SLC calculation scheme. All these different schemes were mainly influenced by the type of the turbomachine the method was applied to (radial or axial), the nature of the flow (subsonic or supersonic) being considered and the level up to which the flow viscosity and circumferential in-homogeneities were taken into account. In many cases, the various calculation schemes were also influenced by the particular characteristics of the cascade, such as hub to tip ratio, lean and sweep angle distributions, etc.

During the many years of development of the SLC method, several excursions from the initially proposed solution scheme were made in many aspects. Firstly, differences can be noticed 
concerning the final form of the SLC equation steaming from the coordinate system selection and the quantities each researcher includes in the solution scheme. For instance, Frost [16] defined a solution grid formed by calculating planes parallel to the upstream and downstream boundaries, whereas Jennious and Stow [19] defined a solution grid by approximating streamlines using a curve fit through points of equal mass flow. Differences can be spotted even in the way the SLC is calculated. Two different approaches are suggested by Shaalan and Dareshyar [20] and Wilkinson [21].

As mentioned previously, the quantities each researcher includes in the final equation have also an impact on the type of the final differential equation. This particular issue is discussed in detail in the third section of this manuscript. It is worth noticing that almost none of the final SLC equations met in various literature texts are the same, although they do have some similarities. Moreover, differences between researchers applying the same method can be found on the solution process depending on whether inter-blade stations are considered and also depending on empirical rules applied to aid the solution process and for controlling the convergence procedure. To cite an example, Casey [22] has considered inter-blade stations, whereas Denton [23] in his very early work did not. Concerning the convergence control, Wilkinson [21] proposed a very interesting study. Finally, another field where different approaches are met is the way each researcher chooses to take into account the presence of viscous forces into the flow, as well as the circumferential variations along the blade span. In some earlier SLC methods those effects were not considered at all. Horlock [24] suggested the inclusion of a non-conservative body force acting opposite to the stream direction in order to make the momentum equation consistent to the in-viscid assumption. Concerning the inclusion of circumferential effects, which are not present in a 2-D method such as the SLC, three models most commonly used have been reviewed by Horlock and March [25]. The models are based on the replacement of the actual cascade with a cascade containing an infinite number of blades, simulating thus the blade action by an axi-symmetric flow with distributed body forces along the blade and by considering the flow on a suitably defined "mean stream surface" [22].

Authors have adopted the 'system approach' in order to derive the REE, for an elementary fluid element moving through the blade rows. The cylindrical coordinates are more appropriate for this study, than any other system of coordinates, given the geometry of a turbomachine. More precisely, the principal directions will be the circumferential, or whirl direction " $w$ ", the meridional direction " $\mathrm{m}$ " and the normal to the meridional direction " $\mathrm{n}$ ". The $\mathrm{m}-\mathrm{n}$ directions are used instead of the axial-radial, because the basic concept of the current method of solution is based on the SLC. In figure 3 the principal and some auxiliary directions are being defined, as well as the angles involved in the derivation process.

The application of Newton's second law of motion, for this elementary fluid element, gives the following system of equations, for the three principal directions [26]:

$$
\mathrm{m}: \mathrm{V}_{\mathrm{m}} \cdot \frac{\mathrm{dV}_{\mathrm{m}}}{\mathrm{dm}}-\frac{\mathrm{V}_{\mathrm{w}}^{2}}{\mathrm{r}} \cdot \sin \varepsilon=-\frac{1}{\mathrm{\rho}} \cdot \frac{\mathrm{dP}}{\mathrm{dm}}+\mathrm{F}_{\mathrm{m}}
$$




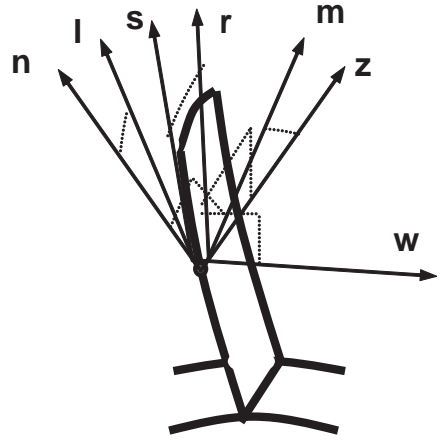

Figure 3. Definition of angles and directions [27]

The first term corresponds to the inertial force due to the acceleration of the element in the meridional direction. The second term denotes the centrifugal force term due to the circumferential movement, projected onto the meridional direction. The third term corresponds to the pressure force in the same direction and the last term represents the body forces exerted onto the element. In the normal direction the equation gives:

$$
\mathrm{n}: \frac{\mathrm{V}_{\mathrm{m}}^{2}}{\mathrm{r}_{\mathrm{c}}}-\frac{\mathrm{V}_{\mathrm{w}}^{2}}{\mathrm{r}} \cdot \cos \varepsilon=-\frac{1}{\mathrm{Q}} \cdot \frac{\mathrm{dP}}{\mathrm{dn}}+\mathrm{F}_{\mathrm{n}}
$$

The first term here corresponds to the centrifugal force due to the movement of the element on a curved streamline. The second term denotes the centrifugal force term due to the circumferential movement of the element projected on the normal direction. The third term corresponds to the pressure forces on the same direction and the forth term represents the body forces exerted onto the fluid element. Finally on the circumferential direction Newton's second law gives:

$$
\mathrm{w}: \frac{\mathrm{V}_{\mathrm{m}}}{\mathrm{r}} \cdot \frac{\mathrm{d}\left(\mathrm{r} \cdot \mathrm{V}_{\mathrm{w}}\right)}{\mathrm{dm}}=\mathrm{F}_{\mathrm{w}}
$$

According to the above equation, the body forces are equal to the corriolis force, since the element moves in the meridional direction, while the whirl velocity component is varying. The above equations are applicable for steady, axi-symmetric in-viscid flow and the final aim is to derive from them a differential equation, the solution of which will give as a result the meridional velocity profile, defined on the m-n plane. Consequently the circumferential flow variations, the flow viscosity and the 3-D nature of the flow, seem to be left out from the analysis. However, this is not entirely true, because some mathematical manipulations were proposed in order to take into account those effects artificially. It is more convenient for the solution process, to have the final equation expressed in s-m coordinates, rather than in n-m coordinates, since the meridional velocity profile is required along the blade leading and 
trailing edges. The variation of pressure along the s direction is given by the following equation that steams out from the state equation:

$$
\frac{1}{Q} \cdot \frac{d P}{d s}=\frac{d H}{d s}-T \cdot \frac{d S}{d s}-\frac{V_{m} \cdot d V_{m}}{d s}-\frac{V_{w} \cdot d V_{w}}{d s}
$$

Equations 3 and 4, involve the pressure variation along the meridional and normal directions respectively. Substituting those equations into equation 6 , the following equation results:

$$
\cos (\varepsilon-\gamma) \cdot\left(F_{n}-\frac{V_{m}^{2}}{r_{c}}+\frac{V_{w}^{2}}{r} \cdot \cos \varepsilon\right)+\sin (\varepsilon-\gamma) \cdot\left(F_{m}-\frac{V_{m} \cdot d V_{m}}{d m}+\frac{V_{w}^{2}}{r} \cdot \sin \varepsilon\right)=\frac{d H}{d s}-T \cdot \frac{D S}{d s}-\frac{V_{m} \cdot d V_{m}}{d s}-\frac{V_{w} \cdot d V_{w}}{d s}
$$

Introducing rothalpy into equation 7 instead of enthalpy, a much more convenient quantity when examining rotating flow, and moving from the absolute system of reference to the relative, by substituting:

$$
\mathrm{V}_{\mathrm{w}}=\mathrm{W}_{\mathrm{w}}+\omega \cdot \mathrm{r}
$$

The following equation appears:

$$
\frac{\mathrm{V}_{\mathrm{m}} \mathrm{dV} \mathrm{V}_{\mathrm{m}}}{\mathrm{ds}}=\sin (\varepsilon-\gamma) \cdot \frac{\mathrm{V}_{\mathrm{m}} \mathrm{dV} \mathrm{V}_{\mathrm{m}}}{\mathrm{dm}}+\cos (\varepsilon-\gamma) \cdot \frac{\mathrm{V}_{\mathrm{m}}^{2}}{\mathrm{r}_{\mathrm{c}}}+\frac{\mathrm{dH}}{\mathrm{ds}}-\mathrm{T} \cdot \frac{\mathrm{dS}}{\mathrm{ds}}-\sin (\varepsilon-\gamma) \cdot \mathrm{F}_{\mathrm{m}}-\cos (\varepsilon-\gamma) \cdot \mathrm{F}_{\mathrm{n}}-\frac{\mathrm{V}_{\mathrm{w}}}{\mathrm{r}} \cdot \frac{\mathrm{d}\left(\mathrm{r} \cdot \mathrm{V}_{\mathrm{w}}\right)}{\mathrm{ds}}
$$

As it was mentioned before, the two main flow effects left out from the equation are the fluid viscosity because the flow is treated as in-viscid and the pressure variation across the blade to blade direction due to the 3-D treatment of the flow. The first effect is represented by a drag force term $F_{D}$ acting on the opposite direction of the flow and the second effect is taken into account by the introduction of a pressure force acting normal to the side surface of the infinitesimal volume. The drag force is related to the loss mechanism of the flow, thus related to the entropy generation in the meridional direction:

$$
F_{D}=-\cos \beta \cdot T \cdot \frac{d s}{d m}
$$

The direction vector of the pressure force according to figure 4 equals:

$$
\frac{\vec{F}_{\mathrm{p}}}{\mathrm{F}_{\mathrm{p}}}=-\sin \beta \cdot \cos \lambda \cdot \dot{\mathrm{i}}_{\mathrm{m}}+\cos \beta \cdot \cos \lambda \cdot \mathrm{j}_{\mathrm{w}}+\left(\frac{\sin \lambda \cdot \cos \beta}{\cos (-\gamma)}+\cos \lambda \cdot \sin \beta \cdot \tan (\varepsilon-\gamma)\right) \cdot \overrightarrow{\mathrm{k}}_{\mathrm{n}}
$$

However, in equation 9 the force terms appearing are lying on the normal and meridional directions. Consequently those force terms should be expressed with respect to the drag and pressure forces and then substituted back into equation 9. Finally, the later force terms should also be expressed with respect to the flow parameters in order to bring the final equation into a form so that it depends only on velocity components, flow and geometrical angles and 
thermodynamical parameters. The drag force is already into such a form in equation 10. As far as the pressure force is concerned the following equation is suggested:

$$
\mathrm{F}_{\mathrm{P}}=\frac{\mathrm{F}_{\mathrm{w}}}{\cos \beta \cdot \cos \lambda}+\frac{\sin \beta}{\cos \lambda} \cdot \mathrm{T} \cdot \frac{\mathrm{ds}}{\mathrm{dm}}
$$

Where Fw according to equations 5 and 7 equals:

$$
\begin{gathered}
\mathrm{F}_{\mathrm{w}}=\frac{\mathrm{V}_{\mathrm{m}}}{\mathrm{r}} \cdot\left(\frac{\mathrm{d}\left(\mathrm{r} \cdot\left(\mathrm{W}_{\mathrm{w}}+\omega \cdot \mathrm{r}\right)\right)}{\mathrm{dm}}\right) \\
\mathrm{F}_{\mathrm{W}}=\frac{\mathrm{V}_{\mathrm{m}}}{\mathrm{r}} \cdot \frac{\mathrm{d}\left(\mathrm{r} \cdot \mathrm{W}_{\mathrm{w}}\right)}{\mathrm{dm}}+2 \cdot \mathrm{V}_{\mathrm{m}} \cdot \omega \cdot \sin \varepsilon
\end{gathered}
$$

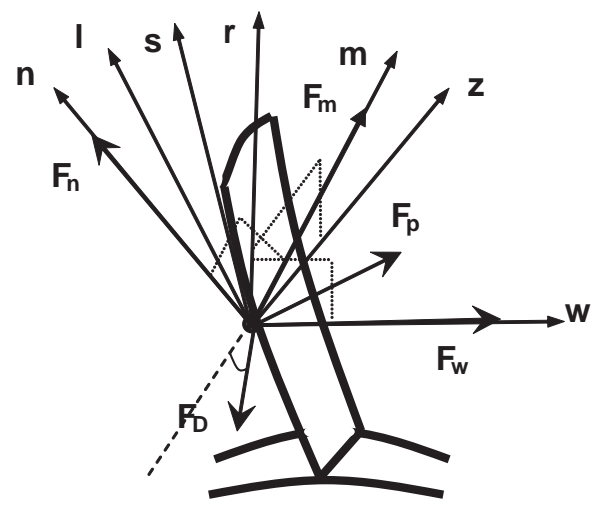

Figure 4. Definition of force vectors [27]

According to figure 4, the force terms $F_{w}$ and $F_{p}$ can be expressed with respect to $F_{m}$ and $F_{n}$ and then substituted back to equation 9 in order to give:

$$
\begin{aligned}
& \frac{\mathrm{V}_{\mathrm{m}} \mathrm{dV}}{\mathrm{ds}}=\sin (\varepsilon-\gamma) \cdot \frac{\mathrm{V}_{\mathrm{m}} \mathrm{dV} \mathrm{V}_{\mathrm{m}}}{\mathrm{dm}}+\cos (\varepsilon-\gamma) \cdot \frac{\mathrm{V}_{\mathrm{m}}^{2}}{\mathrm{r}_{\mathrm{c}}}-\frac{\mathrm{W}_{\mathrm{w}}}{\mathrm{r}} \cdot \frac{\mathrm{d}\left(\mathrm{r} \cdot \mathrm{W}_{\mathrm{w}}\right)}{\mathrm{ds}}-2 \cdot \omega \cdot \mathrm{W}_{\mathrm{w}} \cdot \cos \varepsilon+\frac{\mathrm{dI}}{\mathrm{ds}}-\mathrm{T} \cdot \frac{\mathrm{d} S}{\mathrm{ds}} \\
& -\tan \lambda \cdot\left(\frac{\mathrm{V}_{\mathrm{m}}}{\mathrm{r}} \cdot \frac{\mathrm{d}\left(\mathrm{r} \cdot \mathrm{W}_{\mathrm{w}}\right)}{\mathrm{dm}}+2 \cdot \mathrm{V}_{\mathrm{m}} \cdot \omega \cdot \sin \lambda\right)+\mathrm{T} \cdot \frac{\mathrm{dS}}{\mathrm{ds}} \cdot\left((\sin \varepsilon-\gamma) \cdot \cos ^{2} \beta-\tan \lambda \cdot \sin \beta \cdot \cos \beta\right)
\end{aligned}
$$

The above form of the full REE is found in most relevant texts. It can be used in order to conduct a quick quantitative estimation concerning the flow within turbomachines. The solution of equation 15 will lead to the meridional velocity distribution along the blade leading and trailing edges, or even along inter-blade stations if so defined. However the viscous nature of the flow is not yet fully introduced into calculation. Flow viscosity will generally cause secondary flows and fluid friction against solid surfaces. The aforementioned principal causes 
will lead to several localized phenomena that are independently studied and quantified on the basis of empirical correlations. There are three major areas of irreversibilities:

i. $\quad$ Flow deviations on blade leading and trailing edge. The aim of flow deviation models is to define flow incidence and deviation angles in relation to flow and blade profile parameters.

ii. Boundary layer growth along wetted surfaces. Boundary layer prediction models may end up becoming extremely complex, depending on the level of accuracy one aims to achieve. Care should be taken at this point to balance the computational power consumption against accuracy.

iii. Frictional losses. Calculating blade losses in compressors is an extremely difficult task due to the complex, three-dimensional nature of the flow field. There are several factors that contribute to the generation of losses in a compressor. It would be fair to say that although consensus exists on the end result, which is an increase in entropy and reduction in total pressure, the exact mechanisms through which losses are generated and their complicated interactions have not been completely understood yet. Several researchers have invested their expertise into correlations of parameters which describe the flow in blade passages. Such correlations usually attempt to synthesize the results of many tests into simpler formulae or sets of curves. They are generally averages of test results or their statistical curve fits. The largest limitation of this approach is that the various empirical correlations can not be expected to sufficiently represent every individual compressor design. A very popular approach to blade row total loss prediction was followed by researchers such as Miller [28] and Creveling and Carmody [29]. It is assumed that the total pressure loss of the blade row is the result of the interaction of different loss components, i.e. profile losses, secondary losses and shock losses which are considered to act through independent mechanisms that is the blade mass flow-averaged total loss factor is given as the sum of specific loss terms:

$$
\bar{\omega}_{\text {tot }}=\bar{\omega}_{\text {prof }} \cdot \mathrm{f}_{\operatorname{Re}}+\bar{\omega}_{\text {sh }}+\bar{\omega}_{\text {sec }}
$$

\subsubsection{Multiparallel compressor method}

Circumferential total pressure distortion component is described by dividing the compressor inlet face into a number of "pie" sectors. These sectors are defined by a number of spokes which are intersected at the compressor centerline and they are extended till the compressor outlet casing. An average total pressure value is assigned to each of these sectors.

Multi-parallel compressor method is a common method used to assess the effect of those distortion profile types, on the compressor and gas turbine engine performance. Circumferential total pressure distortion has been a matter of interest for gas turbine engine manufacturers for many years. It was soon realized circumferential total pressure gradients at the compressor inlet face cause degradation on compressor stability margin. This has been 
recognized to be the most pronounced effect of this type of distortion. However, compressor performance is also affected. In the mid-fifties the effects of circumferential total pressure gradients have been examined experimentally [30], on an axial turbojet engine. This work had been among the first of a kind. Durning the seventies a vast amount of experimental work had been conducted at NASA Lewis research center. In the first report [31], the effect of several screen induced total pressure distortions had been determined on a J-85-GE13 turbojet engine. A very simple modeling technique is developed for the assessment of circumferential total pressure distortion effects on compressor performance. The "Parallel Compressor" model suggested by Pearson and McKenzie [33]. This model had been validated experimentally [32], extended to unsteady versions, [34], [35], [36], included into computer codes (GasTurb) and reviewed by almost any circumferential distortion related paper. Hynes and Greitzer [37] have proposed an alternative modeling method capable of assessing both steady and unsteady flow phenomena, caused by circumferential total pressure gradients. It is an analytical solution method, expressed through a set of differential equations that were derived from conservation laws applied on an integrated compression system consisting of the inlet plenum, the compression system, the outlet plenum and the throttle. Plourde and Stenning [38] had also developed a compressor flow model, for assessing the attenuation of circumferential total pressure gradients, within a multi-stage compressor. This model although not referring directly on the performance degradation, provides useful information and understanding of the flow phenomena induced by this type of distortion.

The current method adopts a parallel compressor model the way that is presented in reference [33], extended to incorporate more than two compressor sectors. The parallel compressor model is formed by dividing the compressor into two compressor segments (Figure 5).

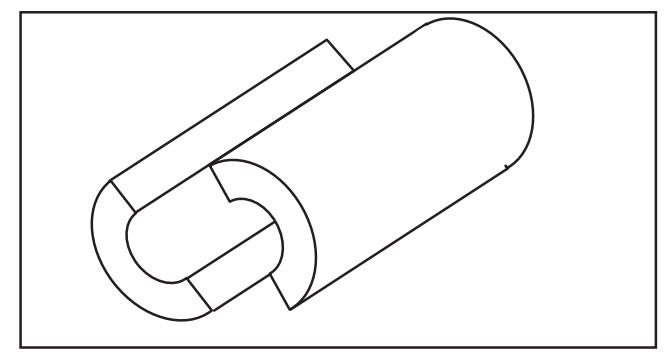

Figure 5. Parallel compressor segments.

Each segment occupies a certain percent of the compressor annulus volume, directly proportional to the angle of extent of each segment. Both segments operate simultaneously and discharge to a common plenum. The first segment is considered to operate under low total inlet pressure and the second is considered to operate under higher inlet total pressure. The fact that both flow regions discharge to a common plenum, justifies the assumption, under certain conditions, that both segments share a common static pressure at their outlet plane. This assumption is valid only if the exit duct is straight and of constant area and the air is 
leaving the compressor under a uniform stator exit angle. If there is a diffuser or a second compressor downstream of the first compressor, then the flow in that region can no longer be considered as 2-D or the exit static pressure as uniform. Implicit in the above description is that: i) two compressors are working in parallel under different inlet total pressure but at a common exit static pressure, ii) no cross flow occurs within a rotor blade row and iii) no flow redistribution takes place within the axial gaps. This is a rather logical assumption to make, because the rotor tip clearance, especially in modern compressors are rather narrow and sometimes actively controlled by external mechanisms and the axial gaps between successive blade rows are when compared to the circumferential length scales, quite small.

Under the above mentioned assumptions each compressor segment can be considered that it is operating on the same non-dimensional compressor speed line, as the "clean" inlet compressor would operate. The "clean" compressor characteristic for high speed compressors should be given as the pressure and temperature ratio versus the non-dimensional mass flow. Each component segment would not theoretically deviate from the non - dimensional speed line, since the compressor characteristic curves were non - dimensionalized with respect both the inlet total boundary conditions and the geometrical characteristics. Figure 6 shows low and high pressure compressor segment operating points on a typical non-dimensional compressor speed line.

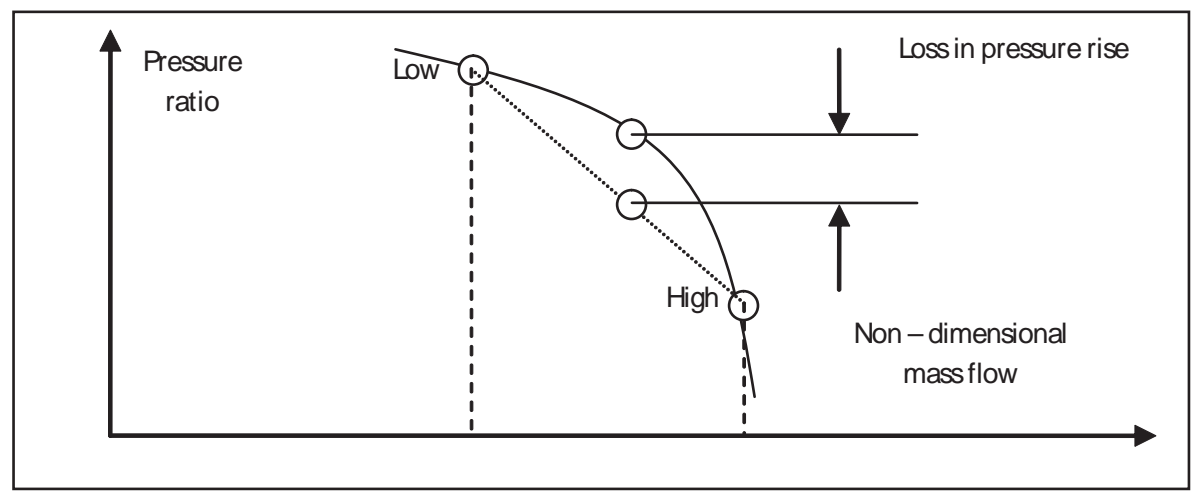

Figure 6. Basic parallel compressor model for compressor response to circumferential total pressure distortion. [39]

Through the parallel compressor model several important aspects of the circumferential inlet total pressure distortion phenomenon can be assessed, at least qualitatively. Firstly as it can be seen on Figure 6, the compressor mean pressure rise is lower than it would have been achieved, if the compressor was operating at the same mean mass flow, uniformly distributed over the compressor inlet face. Also the low inlet total pressure compressor segment produces higher pressure ratio and therefore operates closer to the surge line. Consequently although the compressor average operating point may fall within the stable region of the compressor map, a considerable part of the compressor (the low pressure segment) is working in the unstable region of the map. Consequently flow instability under circumferential total pressure 
distortion may be initiated earlier (at higher mass flows) than it would have, if the compressor was operating under uniform inlet total pressure. The stall criterion that it is used along with parallel compressor model is that stall occurs when the low pressure region operating point, has crossed the stability line. In order to determine the surge line shift under a given circumferential total pressure profile, the operating point of the low pressure compressor segment, is placed on the surge line. The segment's corresponding mass flow is determined after attenuation by the pie sector angle and subsequently it is non-dimensionilized with respect to the corresponding inlet boundary conditions and cross-sectional area. Consequently the total pressure and temperature rise of the segment are read directly by the "clean" compressor performance maps.

\subsubsection{Streamline curvature software description}

The SLC based flow simulation software obeys to the architecture mentioned in paragraph 5.1.2. The pre-processing unit having received all necessary geometrical inputs, defines the compressor geometry and sets the initial position of the streamlines. The processing unit upon convergence, will have defined the flow-field and the distribution of all thermodynamic parameters through the compressor operating range. Given the nature of SLC method calculations being highly iterative, the processor incorporates very sophisticated convergence guidance subroutines. Also several alternatives regarding the consideration of viscous phenomena are offered to the user. However it is beyond the scope of this chapter to describe the details of loss models and convergence schemes. There are several publications that the reader could refer to, shall he/she is further interested in the specific field.

The post processing module of a through flow software such as the SLC, would ideally include the streamline visualization through the compressor and preferably the performance map. Options for comparative performance graphs under various inlet conditions and several different geometries turn out to be very informative and useful especially for educational purposes.

The stand alone streamline curvature software SOCRATES has evolved over the past years to handle transonic flow regimes and chocking conditions. A graphical user interface is also under development giving great flexibility to the user regarding compressor geometry definition, loss model selection etc.

\subsubsection{Extended compressor in parallel" software description}

The "extended compressor in parallel" model is not programmed as an entirely separate code, since it is a straight forward, simple calculation. It is embedded as a subroutine into the 0-D gas turbine engine performance code presented in the following section. The input data is: i) the number of compressor inlet face segments, ii) the angle of extent of each segment, iii) the average inlet total pressure and temperature of each segment and iv) the compressor inlet and outlet cross-sectional area of each segment.

The processing unit houses the calculation procedure which is completed into three concentric iterative loops. The outer most calculation loop is there to repeat the calculations for all possible 
settings of the variable inlet guide and stator vane angles. The second iterative loop is set to repeat the calculations performed for a single compressor speed line and the third loop is set to establish the exit static pressure balance for all the compressor segments specified in the input file. Finally there is not much post - processing that can be done to the results, other than demonstrating the dislocation of the surge line depending on the inlet distorted temperature and pressure profile.

\subsection{Hot engine section}

\subsubsection{Zero dimensional analysis}

The 0-D analysis is an integral type of analysis in the sense that the individual engine components are considered as "black boxes'. Details of the flow within the engine components do not influence the result. Prerequisite for the application of the method are the engine components' performance, in terms of non-dimensional performance maps or alternatively by means of empirical correlations, depending on the information available. The main output is the engine performance for a given handle setting. In practice apart from the engine mass flow, in order to determine the overall engine performance, total thermodynamic quantities (such as total pressure and total temperature) and exit velocity are sufficient.

The intake performance under the assumption that the flow in not subjected to any kind of heat exchange with the environment, is fully determined as soon as the pressure recovery factor is determined. The pressure recovery factor can be read from an intake performance map where it is plotted against various intake inlet Mach numbers. Alternatively it can be calculated through an empirical correlation or it can be input directly as a value emerging purely from experience.

For the compressor, the performance map is usually plotted as the pressure ratio against the non dimensional air flow along lines of constant non-dimensional or relative rotational speed. Constant isentropic or adiabatic efficiency contours appear on the same graph.

Turbine's performance map is usually plotted as the expansion ratio or non-dimensional enthalpy drop versus the non-dimensional mass flow for constant non-dimensional rotational speed lines. The efficiency is usually plotted on a separate graph against the same parameters (non-dimensional mass flow and non dimensional rotational speed). A typical nozzle performance map includes the flow and discharge pressure coefficients as a function of the pressure ratio.

Finally concerning the combustion chamber, many kind of maps may be used, for the determination of the exit total temperature, the fuel flow (one of the previous two parameters is usually given as the engine handle) and the fuel flow composition. Similarly, total pressure loss can also be determined through performance maps or alternatively by the use of an empirical correlation based on hot and cold pressure loss coefficients.

The aim of the 0-D analysis is to fully determine pressures and temperatures as well as the other engine performance parameters throughout the engine, under any given set of boundary conditions. The solution is carried out at a certain off-design point which is specified by a user 
defined value of an appropriate engine handle. Engine handles can be the fuel flow, the rotational speed, or the turbine inlet temperature. The solution scheme, making use of the conservation of mass, energy and power, is searching for the set of operating points on each component's performance map with the aim of achieving the mechanical and thermodynamical engine matching.

At any given flight condition the Mach number and the altitude will determine the airflow at the inlet plane of the first compressor in the row. Subsequently, based upon the compressor's rotational speed and pressure ratio which are fixed once the operating point is fixed on the performance characteristic map, the compressor may not be capable of passing the amount of airflow coming from the intake duct. This gives rise to a flow imbalance. The same holds for all engine's compressors installed downstream. Similarly for the engine combustion chamber, given the burner exit temperature or fuel flow the exit pressure, the airflow at the first turbine face can be determined. The turbine may or may not be able to pass this airflow, depending on its operating point. In a similar way, the same flow imbalances may rise to all turbines and nozzles downstream. Apart from the flow imbalances, mechanical power imbalances may also exist, between a turbine's generated power and the coupled compressor absorbed power, for instance.

All these imbalances are treated as errors. The number of these errors varies according to the engine type. In any case a certain number of equations emerging from the conservation laws are needed for the engine to be balanced mechanically and thermodynamically. Various 0-D solution schemes can be employed for the error minimization. The error minimization process is realized by shifting the operating points on the components performance maps. There are three types of iterative solution schemes that can be used:

- The concentric iterative solution scheme.

- The crossover iterative solution scheme.

- The simultaneous iterative solution scheme.

According to the first two solution schemes the error appearing after the application of a certain conservation law, is minimized by changing a certain engine parameter. According to the third solution scheme, all errors are minimized simultaneously by changing simultaneously a prespecified set of engine parameters. From the mathematical point of view the first two methods are much simpler. A simple trial and error iterative solution scheme for each air flow and power imbalance is enough. For the third method a more complicated mathematical solution scheme is required, like for instance a multi-dimensional Newton-Raphson method. The simultaneous iteration however is more stable than the previous two but higher computational power is required.

The current simulation program is based on the first type of iterative method, the concentric iteration. The operation of a single spool turbojet engine is simulated under any given set of boundary conditions. The engine rotational speed is set to be the engine handle, the parameter that is employed by the user to set the off-design condition. 


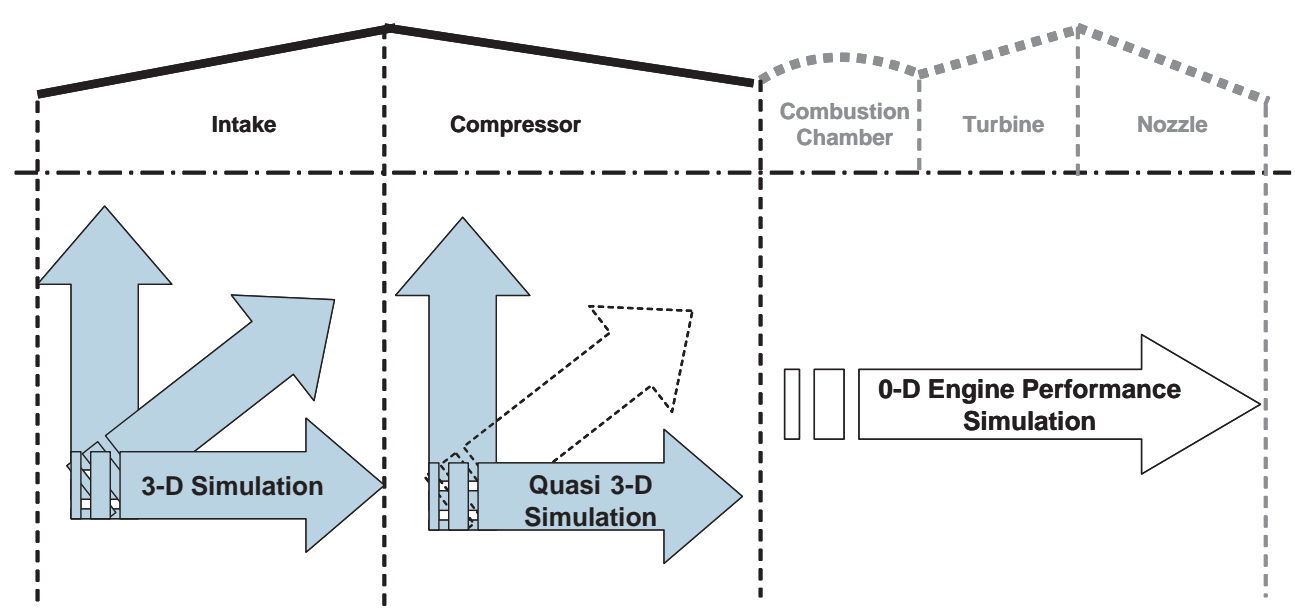

Figure 7. Intake - Gas Turbine Variable Dimension Performance Simulation Method.

\section{Gas turbine engine simulation}

\subsection{Intake - Gas turbine variable dimension performance simulation method}

As it was mentioned in the introductory chapter a single calculation method whichever that could be, cannot cover the need for a "light", in terms of computational load, gas turbine engine performance simulation code, able of addressing engine operation under non-uniform inlet conditions. A synthesis of several computational methods is therefore inevitable. The structural elements of the synthesized calculation method have already been described in the preceding corresponding sections.

Given the fact that flow within the intake and mostly within the compressor is redistributed any type of pressure or temperature distortion at the intake inlet practically vanishes by the time the working medium enters the combustion chamber. Even if any pressure or temperature gradients are persisting till that engine station, given the intense mixing that takes place within the combustion chamber, gradients of thermodynamical properties are redefined on a completely new basis, the combustion process. Consequently a 0-D analysis method downstream of the compressor is sufficient. Generally speaking the use of 2-D or 3-D flow simulation methods and their derived tools at hot section of the engine could only be justified in the case of detailed design.

On the contrary, the performance of the cold engine section, namely the intake and the compressor, is affected by the uneven flow properties distribution at the intake inlet. Regarding the intake section, the flow remains three dimensional and a 3-D flow simulation tool should be used in order to convey the flow information down to the compressor. Despite the fact flow non-uniformities are to a certain extent attenuated until they reach the compressor section, 
applying a 0-D or an 1-D flow simulation method will discard most of the information and will lead to highly inaccurate results.

\subsection{Proposed variable dimension simulation}

The current simulation technique was built with the aim of embracing the combined - coupled performance of the intake and the compressor. In reality the compressor map that comes as an intermediate output of the intake - compressor simulation, represents not only the compressor performance over its operating range as it is usually seen, but it also includes indirectly the intake performance. The simulation focuses on a certain compressor operating point defined by a certain rotational speed value and a mass flow value falling in the range of the selected rotational speed. Once the intake simulation has converged under a certain inlet boundary condition set, the flow-field on the compressor inlet plane is practically defined. 3D flow calculation methods for compressor flow simulation have all been excluded mainly due to their high computational resources demand. In other words the derived compressor inlet boundary conditions have to be decomposed in order to reduce their dimensions. Two components are considered:

i. Variation along the radial direction

ii. Variation along the circumferential direction.

The derived engine flow simulation tool was designed with the intention of assessing the impact of 3-D pressure profiles on the engine performance. Therefore the distinction was made on the basis of the effect that each component has on compressor performance. The main effect of circumferential total pressure distortion on compressor performance is the surge line shift. On the contrary radial pressure distortion has the exact opposite effect, especially for multistage compressors. According to a NASA report [30] radial distortion did not appear to change the flow in the stages that control stall, because of the rapid attenuation of the distortion within the compressor. In other words surge line position is not severely affected by this type of distortion. Based on the above observations, the SLC code under the radial profile of boundary conditions, computes the pressure ratio and the isentropic efficiency corresponding to the initially specified mass flow and rotational speed. While the same rotational speed is retained, a small (positive of negative) increment is added to the mass flow and the whole process starting from the intake simulation, is repeated again until the entire mass flow range for this rotational speed is covered. Subsequently a small increment (positive or negative) is added to the rotational speed and the whole process is repeated again until a certain range of non dimensional speed lines is covered. By the end of the above described process the full compressor - intake performance characteristic map will have been obtained. The compressor map as extracted from the SLC code is fed as an input to the "extended compressor in parallel" code. The number of circumferential sectors, their extent, as well as their average pressure, should be specified as an input. The exact number of sectors selected in order for the circumferentially distorted pattern be described in best, should ideally be defined through a sensitivity analysis. Given the above input, the surge line shifts will be predicted by the "extended compressor in parallel" code. At this point the performance of the intake - compressor is fully 
determined. The resulted compressor performance map, together with the input data referring to the remaining engine components, is fed into the $0-\mathrm{D}$ simulation code for the engine performance to be defined.

On figure 8 it is demonstrated on the compressor map, a snapshot of the response of a small single spool turbojet engine operating behind a generic intake under certain sets of nonuniform inlet conditions.

$20 \%$ Tip Deficit Radial Distortion + 180 Deg-20\% Intensity Circumferential Distortion

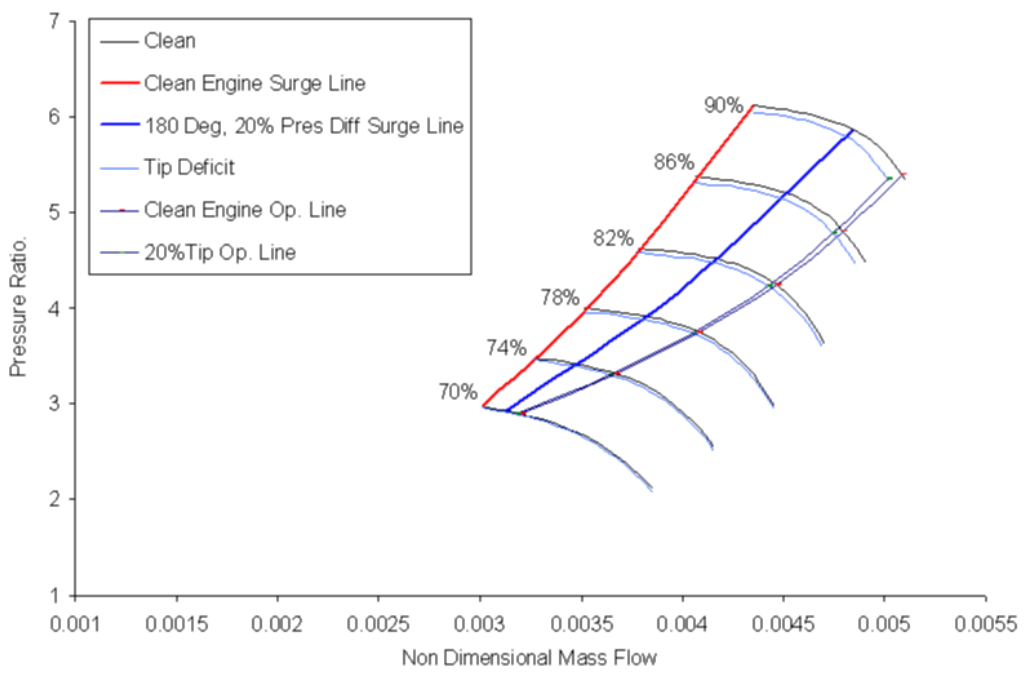

Figure 8. Compressor map shifts under various inlet conditions.

\section{Closure}

Gas turbine engine performance simulation is a very wide research sector of gas turbine related technology. The current chapter focused on the intermediate fidelity - relative low computational power consumption type of methods and derived tools. These tools in order to meet the contradictive requirements for 3-D flow treatment and fast convergence are based on more than one method. Moreover they make use of stored performance related information that come out from experiment and/or high fidelity flow simulation. Such simulation tools prove very convenient to gas turbine engine manufacturers as they can "multiply" the value of their numerous stored experimental data. It is the author's view that such hybrid simulation methods will on one hand be gaining increasing attention by the manufacturers, while on the other hand they consist a brilliant research field for creative thinking a combination which will lead to very valuable applications in the future. 


\section{Nomenclature}

Abbreviations

CFD - Computational Fluid Dynamics

RANS - Reynolds Averaged Navier Stokes

REE - Radial Equilibrium Equation

SLC - Streamline Curvature

VLM - Vortex Lattice Method

0-D - Zero-Dimensional

1-D - One-Dimensional

2-D - Two-Dimensional

3-D - Three-Dimensional

Symbols

F - Force

H - Enthalpy

I - Rothalpy

P - Pressure

S - Entropy

$\mathrm{T}$ - Temperature

$\mathrm{V}$ - Absolute air velocity

W - Relative velocity

c - Constant of integration

$\mathrm{i}, \mathrm{j}, \mathrm{k}$ - Unit vectors

$\mathrm{m}$ - Meridional direction

$\mathrm{n}$ - Normal (to the meridional) direction

$\mathrm{r}$ - Radius, radial direction

rc - Radius of curvature

s - Tangential along the blade edge, direction

z - Axial direction

Greek Symbols 
$\Gamma$ - Circulation

$\alpha$ - Absolute flow angle

$\beta$ - Relative flow angle

$\gamma$ - Sweep angle

$\varepsilon$ - streamline slope angle

$\lambda$ - Lean angle

@ - Density

$\omega$ - Angular speed, Loss factor

Subscripts

D - Drag

P - Pressure

Re - Reynolds

j - Streamline counter

m - Meridional direction

$\mathrm{n}$ - Normal direction

prof - Profile

r - Radial direction

sec - Secondary

sh - Shock

tot - Total

w - Whirl direction

z - Axial direction

\section{Author details}

Ioannis Templalexis*

Hellenic Air Force Academy Department of Aeronautical Sciences Section of Thermodynamics, Propulsion and Power Systems, Greece 


\section{References}

[1] www.gasturb.de

[2] M.G. Turner, J.A. Reed, R. Ryder and J.P. Veres, "Multi-Fidelity Simulation of a Turbofan Engine with Results Zoomed into Mini-Maps for a Zero-D Cycle Simulation," ASME Paper No. GT2004-53956, Vienna, Austria, June, 2004 (Also NASA TM-2004-213076)

[3] Alexiou A., Baalbergen E., Mathioudakis K., Kogenhop O., Arendsen P., "Advanced Capabilities for Gas Turbine Engine Performance Simulations", ASME paper GT2007-27086.

[4] Alexiou A., Mathioudakis K., "Gas Turbine Engine Performance Model Applications Using An Object-Oriented Simulation Tool", ASME paper GT2006-90339.

[5] Alan Hale, Milt Davis, and Jim Sirbaugh, "A Numerical Simulation Capability for Analysis of Aircraft Inlet-Engine Compatibility" J. Eng. Gas Turbines Power 128, 473 (2006)

[6] Pachidis, V., Pilidis, P., Talhouarn, F., Kalfas, A. and Templalexis, I., "A fully integrated approach to component zooming using computational fluid dynamics", Transactions of the ASME, Journal of Engineering for Gas Turbines and Power, Vol. 128, No.3, p. 579, July 2006.

[7] Pachidis, V., Pilidis, P., Marinai, L., Templalexis, I., “Towards a full two dimensional gas turbine performance simulation", Proceedings of the RASoc, The Aeronautical Journal, AJ-3127, June 2007.

[8] Templalexis, I., Pilidis, P., Pachidis, V. and Kotsiopoulos, P., “Development of a 2D compressor streamline curvature code", Transactions of the ASME, Journal of Turbomachinery, TURBO-06-1178, Vol. 129, Issue 4, October 2007.

[9] [136] Veres J.P. Overview Of High Fidelity Modelling Activities in The Numerical Propulsion System Simulations Project, NASA TM 2002-211351.

[10] Panel Methods an Introduction, NASA Technical Paper 2995, Decmber 1990.

[11] Katz, J. Plotkin A. Low Speed Aerodynamics From Wing Theory To Panel Methods. Mc Graw Hill Series in Aeronautical and Aerospace Engineering, 1989.

[12] Wu, C.H., 1952, “A General Theory of Three Dimensional Flow in Subsonic and Supersonic Turbomachines of Axial, Radial and Mixed Flow", NACA TN-2604.

[13] Smith, L.H. 1966, "The Radial Equilibrium Equation of Turbomachinery", Trans. A.S.M.E., Series A, Vol 88.

[14] Novak, R.A. "Streamline Curvature Computing Procedures for Fluid Flow Problems.", A.S.M.E. paper 66-WA/GT-3. 
[15] Silvester, M.E. and Hetherington, R., 1966, "Three Dimensional Compressible Flow Through Axial Flow Turbomachines." Numerical Analysis an Introduction, Academic Press.

[16] Frost, D.H., 1972, “A Streamline Curvature Through-Flow Computer Program for Analysing the Flow Through Axial-Flow Turbomachines.", Aeronautical Research Council, R\&M 3687.

[17] Senoo, Y. and Nakase, Y., 1972 "An Analysis of Flow Through a Mixed Flow Impeller.", ASME J. of Eng. for Power, pp. 43-50.

[18] Novak, R.A. and Hearsey, R.M., 1977, "A Nearly Three-Dimensional Intrablade Computing System for Turbomachinery.", J. of Fluids Eng. pp. 154-166.

[19] I.K. Jennions, P. Stow, 1985, “A Quasi-Three-Dimentional Turbomachinery Blade Desigh System: Part I - Throughflow Analysis.”, J. of Eng. for Gas Turbines and Power, Vol. 107, pp. 301-307.

[20] Shaalan, M. R. A. and Daneshyar, H., 1972, "A Critical Assessment of Methods of Calculating Slope and Curvature of Streamlines in Fluid Flow Problems." Proc. of the Institution of Mechanical Engineers, Vol. 186.

[21] Wilkinson, D. H., 1969-70, "Stability, Convergence and Accuracy of 2-D Streamline Curvature Methods Using Quasi-Orthogonals." Proc. of the Institution of Mechanical Engineers, Vol. 184.

[22] M.V. Casey, 1984, "A Streamline Curvature Throughflow Method for Radial Turbocompressors.", C57/84 IMechE.

[23] Denton, J. D., 1978, “ThroughFlow Calculations for Transonic Axial Flow Turbines.”, J. of Eng. for Power, Vol.100, pp. 212-218.

[24] Horlock, J. H., 1971, “On entropy production in adiabatic flow in Turbomachines", ASME Paper No 71-F3-3.

[25] Horlock, J. H. and March, H., "Flow models for turbomachines.", Journal of Mechanical Engineering.

[26] Arthur, J. Wennerstorm, 2000, “Design of Highly Loaded Axial Flow Fans and Compressors", Consepts ETI Press, pp93-99.

[27] Templalexis, I., Pachidis, V., Pilidis, P., and Kotsiopoulos, P., “The Effect of blade Lean on the Solution of the Full Radial Equilibrium Equation", GT2008-50259, ASME Turbo Expo, Power For Land, Sea and Air, Berlin, Germany, June 2008.

[28] Miller G. R., Lewis, G. W. and Hartmann M. J., 1961 “Shock Losses In Transonic Rotor Rows", Transactions of the ASME, Journal of Engineering for Power, Vol. 83, pages 235-242. 
[29] Creveling H. F. and Carmody R. H., 1968 “Axial Flow Compressor Computer Program for Calculating Off-Design Performance (Program IV)”, General Motors, Allison Division, Indianapolis, Prepared for NASA, Report CR-72427.

[30] Hager, R., D. 1977, Analysis Of Internal Flow Of J85-13 Multistage Compressor. NASA TM X-3513, Washington June 3, 1955.

[31] Calogeras, J., E., Johnsen, R., L., Burstadt, P., L., “Effect Of Screen Induced Total Pressure Distortion On Axial Flow Compressor Stability." NASA TM X-3017. Lewis research center, National aeronautics and space administration, May 1974.

[32] Milner, E., J., 1977, Performance And Stability Of A J85-13 Compressor With Distorted Inlet Flow., NASA TM X-3515. Lewis research center, National aeronautics and space administration, May 1977.

[33] Pearson H., McKenzie, A., B., Wakes In Axial Compressors. J. R. Aeronautical Society, Vol 63, No 583 July 1959, pp.415-416.

[34] Mazzawy, R., S., Multiple Segment Parallel Compressor Model For Circumferential Flow Distortion. ASME Journal of engineering for power, Vol 99, Apr.1977, pp228-246.

[35] Steenken, W., G., Modeling Compressor Component Stability Characteristics-Effects Of Inlet Distortion And Fan Bypass Duct Disturbances. Engine handling, AGARD CP-324, Oct. 1982.

[36] Baghdadi, S. and Lucke, J., E., Compressor Stability Analysis, ASME Paper No. 81WA/FE-18.

[37] Hynes, T., P., Greitzer, E., M., A Method For Assessing Effects Of Circumferential Flow Distortion On Compressor Stability, Journal of turbomachinery, Vol. 109, July 1987, pp.371-379.

[38] Plourde, G., A., Stenning A., H., The Attenuation Of Circumferential Inlet Distortion In Multi-Stage Axial Compressors. AIAA 3rd Propulsion joint specialist conference, Washington, D., C., July 17-21, 1967.

[39] Longley, J. P., Greitzer, E., M., Inlet Distortion Effects In Aircraft Propulsion System Integration. Trans of ASME ASME/94-GT-220, 1994. 
